\title{
Article
}

\section{Temperature Dependence of Dimerization and Dewetting of Large-Scale Hydrophobes: A Molecular Dynamics Study}

Ronen Zangi, and B. J. Berne

J. Phys. Chem. B, 2008, 112 (29), 8634-8644 • DOI: 10.1021/jp802135c • Publication Date (Web): 26 June 2008

Downloaded from http://pubs.acs.org on November 18, 2008

\section{More About This Article}

Additional resources and features associated with this article are available within the HTML version:

- $\quad$ Supporting Information

- $\quad$ Access to high resolution figures

- $\quad$ Links to articles and content related to this article

- $\quad$ Copyright permission to reproduce figures and/or text from this article

\section{View the Full Text HTML}




\title{
Temperature Dependence of Dimerization and Dewetting of Large-Scale Hydrophobes: A Molecular Dynamics Study
}

\author{
Ronen Zangi and B. J. Berne* \\ Department of Chemistry and Center for Biomolecular Simulations, Columbia University, 3000 Broadway, \\ New York, New York 10027
}

Received: March 11, 2008; Revised Manuscript Received: April 28, 2008

\begin{abstract}
We studied by molecular dynamics simulations the temperature dependence of hydrophobic association and drying transition of large-scale solutes. Similar to the behavior of small solutes, we found the association process to be characterized by a large negative heat capacity change. The origin of this large change in heat capacity is the high fragility of hydrogen bonds between water molecules at the interface with hydrophobic solutes; an increase in temperature breaks more hydrogen bonds at the interface than in the bulk. With increasing temperature, both entropy and enthalpy changes for association strongly decrease, while the change in free energy weakly varies, exhibiting a small minimum at high temperatures. At around $T=T_{\mathrm{s}}=360 \mathrm{~K}$, the change in entropy is zero, a behavior similar to the solvation of small nonpolar solutes. Unexpectedly, we find that at $T_{\mathrm{s}}$, there is still a substantial orientational ordering of the interfacial water molecules relative to the bulk. Nevertheless, at this point, the change in entropy vanishes due to a compensating contribution of translational entropy. Thus, at $T_{\mathrm{s}}$, there is rotational order and translational disorder of the interfacial water relative to bulk water. In addition, we studied the temperature dependence of the drying-wetting transition. By calculating the contact angle of water on the hydrophobic surface at different temperatures, we compared the critical distance observed in the simulations with the critical distance predicted by macroscopic theory. Although the deviations of the predicted from the observed values are very small $(8-23 \%)$, there seems to be an increase in the deviations with an increase in temperature. We suggest that these deviations emerge due to increased fluctuations, characterizing finite systems, as the temperature increases.
\end{abstract}

\section{Introduction}

One of the most important manifestations of the hydrophobic effect is the low solubility of nonpolar molecules in water; oil and water do not mix. The phenomenon is often attributed to the relatively strong water-water interaction energy in comparison to the weak solute-water interaction. Therefore, it is expected that the enthalpy change, $\Delta H$, of mixing oil and water will be positive and large. However, experiments on the hydration of small nonpolar solutes around room temperature have shown that $\Delta H$ is small and sometimes even negative. ${ }^{1}$ What then opposes solvation is the large negative unitary entropy change (unitary entropy is the measured entropy minus the mixing entropy), $\Delta S_{\mathrm{u}}$, when nonpolar solutes are introduced into water. $^{2-6}$ Thus, at room temperature, hydrophobic interactions are driven by entropy. A large negative value of $\Delta S_{\mathrm{u}}$ is one of the two characteristics distinguishing hydrophobic solvation from normal solvation (where $\Delta S_{\mathrm{u}}$ is near zero). The second characteristic of hydrophobic solvation is the large and positive heat capacity change, $\Delta C_{\mathrm{p}}$, accompanying the solvation process, ${ }^{4,7-10}$ as opposed to small changes for normal solvation. This indicates that the enthalpy and entropy changes are strong functions of temperature. Both functions increase with temperature, with the consequence that at high temperatures, $\Delta S_{\mathrm{u}}$ approaches zero and $\Delta H$ is large and positive. This means that at high temperatures, the hydrophobic interactions are driven by enthalpy. Measurements have shown that the free energy change, $\Delta G$, for solvating small nonpolar solutes in water is large and positive and very weakly temperature dependent. ${ }^{1,4,11,12}$

* Corresponding author. E-mail: bb8@columbia.edu.
It is a convex function with a maximum at high temperatures that decreases with the size of the solute. ${ }^{13,14}$ (Note that it is $\triangle G / R T$ that corresponds directly to solubility, partition coefficient, or Boltzmann population.) The weak dependency of $\Delta G$ on temperature indicates that the large changes in the enthalpic and entropic contributions with increasing temperature almost completely cancel each other. This unique temperature behavior of thermodynamic functions for transferring nonpolar solutes into aqueous solution is what defines hydrophobicity. ${ }^{15,16}$

A possible explantion for the large negative value of $\Delta S_{\mathrm{u}}$ for the solvation of nonpolar solutes in water around room temperature was first offered by Butler ${ }^{17}$ and Eley ${ }^{8,9}$ and more emphatically stated by Frank and Evans, ${ }^{18}$ who proposed that water molecules around hydrophobic solutes arrange themselves in a quasi-crystalline structure (referred to as an iceberg) in which there is less randomness. These ordered water molecules have an entropy that is lower than that of water molecules in the bulk. If $\Delta S_{\mathrm{u}}$ and $\Delta C_{\mathrm{p}}$ of hydrophobic solvation are functions of the number of water molecules surrounding the solutes, and if the heat capacity change is independent of temperature, then $\Delta S_{\mathrm{u}}$ should vanish at the same point for all hydrophobic solutes. ${ }^{19}$ Indeed, calorimetric data of the solvation of gaseous and liquid hydrocarbons in water show ${ }^{20}$ that $\Delta S_{\mathrm{u}}$ of the transfer process becomes zero at a common temperature, $T=T_{\mathrm{s}}$, around $383 \mathrm{~K}$. It is tempting to assume that at $T_{\mathrm{s}}$ the water molecules around the nonpolar solutes are no longer ordered (relative to the bulk). This assumption implies that hydrophobic solvation at $T_{\mathrm{s}}$ is identical to normal solvation. ${ }^{11,21}$ However, this was contested $^{15}$ by the argument that the heat capacity change 
remains large ${ }^{3}$ at $T_{\mathrm{s}}$ and is very weakly temperature dependent up to $355 \mathrm{~K}$.

What is the molecular picture behind the large and positive $\Delta C_{\mathrm{p}}$ characterizing hydrophobic solvation? On the basis of the observation that (in addition to $\Delta S$ ) the heat capacity change is linearly proportional to the surface area of the nonpolar solute, the following view has been suggested., $811,15,22-24$ At low temperatures, the ordered water molecules around the solute populate low energy, low entropy states. With increasing temperature, the waters surrounding the solute increasingly populate higher energy, higher entropy states. Thus, at high temperatures, the ordered water structure melts. These two energetic states provide an energy storage mechanism, and as a result, the capacity of the solution to absorb heat is large.

The behavior of hydrophobic interactions has been shown to be length-scale dependent. ${ }^{25}$ The changes with length scale arise from the fact that around small solutes, the water molecules can maintain a hydrogen bond network by forming small distortions of hydrogen bonds that accommodate the solute. ${ }^{26}$ However, a distorted hydrogen bond network cannot be maintained in the water layer next to an extended hydrophobic surface, and the hydrogen bond network is then disrupted. ${ }^{25}$ The crossover length between small- and large-scale hydrophobic solvation is $\sim 1 \mathrm{~nm}$. This effect has three important, lengthscale dependent, consequences. The first is the negative value of $\Delta H$ for solvating small hydrocarbon solutes. ${ }^{1}$ It decreases, however, in magnitude for larger hydrocarbons and is close to zero for benzene. ${ }^{22}$ Not surprisingly, for large hydrophobic plates, $\Delta H$ is large and positive. ${ }^{27,28}$ The second consequence is manifested by the instability of liquid water confined between two large hydrophobic plates; if the distance between the plates is small enough, a drying transition is observed, ${ }^{29,30}$ and a microscopic theory for this was proposed. ${ }^{31-33}$ In addition, it has been argued that the interface around an extended single hydrophobic surface is characterized by the depletion of water next to the hydrophobe. ${ }^{29,30}$ The third consequence is related to changes in the magnitude of the hydrophobic interaction in the presence of salts. ${ }^{27,28,34}$ Recent simulations have shown that the effective interactions between large hydrophobic plates are weakened in the presence of salts with a low ionic charge density. On the other hand, the effective interaction between small hydrophobic solutes can be weakened or strengthened depending on the concentration of salt in the aqueous solution. ${ }^{28,34}$

It is of interest that the unfolding reactions of different proteins display certain properties that are common to the solvation of hydrophobic solutes: (i) the unfolding enthalpy is small at room temperature but increases rapidly with temperature, becoming large at high temperatures. ${ }^{11,35}$ (ii) The difference in the heat capacity change for unfolding is independent of temperature and linearly related to the fraction of hydrophobic residues. ${ }^{20,36}$ (iii) Plots of the specific (per unit mass or residue) entropy change for unfolding different proteins intersect at a common temperature. ${ }^{11,37}$ Baldwin demonstrated ${ }^{14,20}$ that this temperature is the temperature $T_{\mathrm{s}}$ at which the contribution to the entropy change from the solvation of hydrophobic residues buried in the folded protein is zero. The residual entropy is probably due to changes in the configurational entropy and solvation of polar groups. These observations provide some of the strongest evidence that hydrophobicity is the major force driving proteins to fold. ${ }^{22}$

Given the difference in the way water solvates large and small nonpolar solutes, it is unclear as to what to expect for the behavior of large-scale hydrophobic interactions as a function of temperature. Using the Lum-Chandler-Weeks (LCW) theory, ${ }^{31}$ it was shown that the excess chemical potential for creating a spherical bubble with a large radius (larger than 1 $\mathrm{nm}$ ) decreases monotonically with temperature. ${ }^{38}$ This was obtained by using experimental values of the energy, isothermal compressibility, and surface tension of liquid water at different temperatures as input to the LCW theory. The results indicate that the temperature dependence of the excess chemical potential is the same as that observed for the liquid-vapor surface tension of water. Since the curve for chemical potential does not display an extremum, it was argued that the entropy change for largescale hydrophobicity does not pass through zero but remains positive and is roughly temperature independent. The nonvanishing positive value of $\Delta S$ was used to explain the scattered values of $T_{\mathrm{s}}$ found in the analysis of a large set of proteins. ${ }^{39}$

As mentioned before, one of the qualitative differences between small- and large-scale hydrophobicity in a strongly hydrophobic system is the occurrence of a drying transition. For interplate distances smaller than a critical value, $D_{\mathrm{c}}$, the confined water is thermodynamically unstable in its liquid phase and evaporates. ${ }^{29}$ This is the drying transition that was predicted between solvophobic particles ${ }^{40,41}$ and demonstrated to exist between hydrophobic plates in water. ${ }^{29}$ However, above a critical value of the plate-water attractive interactions ${ }^{42}$ or below a critical value of the size of the hydrophobic surface, ${ }^{43}$ the drying transition does not take place. These factors, which were studied at room temperature, have recently been shown to play a crucial role in the way multidomain proteins self-associate; the existence of a dewetting transition was found to depend on the geometry and chemical composition of the interacting regions. ${ }^{44,45}$

The prediction of the critical distance, $D_{\mathrm{c}}$, for the drying transition can be calculated using a simple macroscopic analysis. ${ }^{43,46-48}$ It is achieved by equating the expression of the thermodynamic grand potential for water (between the two plates) in the liquid state to that in the vapor state. By assuming a cylindrical shape for the vapor phase, and using Young's equation, the following relation is obtained:

$$
D_{\mathrm{c}}=\frac{-2 \gamma_{1 \mathrm{v}} \cos \theta_{\mathrm{c}}}{\left(P-P_{\mathrm{v}}\right)+2 \gamma_{1 \mathrm{v}} / R_{\mathrm{m}}}
$$

where $\gamma_{\text {lv }}$ is the liquid/vapor surface tension for water, $\theta_{\mathrm{c}}$ is the contact angle of water on the hydrophobic plate, $P$ is the pressure of the liquid (water), $P_{\mathrm{v}}$ is the vapor pressure of water, and $R_{\mathrm{m}}$ is the radius of the disk-like plates. For plates of nanoscale size, the term in the denominator $\left(P-P_{\mathrm{v}}\right)$ is much smaller than $2 \gamma_{1 \mathrm{v}} /$ $R_{\mathrm{m}}$ and can be ignored, yielding

$$
D_{\mathrm{c}} \approx-R_{\mathrm{m}} \cos \theta_{\mathrm{c}}
$$

Thus, for plates of fixed size, the critical distance for drying depends on the contact angle of water on the hydrophobic plates.

Owing to the unique temperature behavior observed for smallscale hydrophobic interactions, we investigated in this study the temperature dependence of thermodynamic functions for the association of large-scale hydrophobic plates. We found that at room temperature the association process is driven both by enthalpy and by entropy. Similar to the small-scale regime, the association process is characterized by a large negative heat capacity change. The origin of this large change in heat capacity is the high fragility of hydrogen bonds between the water molecules at the interface of hydrophobic solutes. The (unitary) entropy and enthalpy change for association strongly depends on temperature, while the change in free energy exhibits a very weak temperature dependence. At around $T=T_{\mathrm{s}}=360 \mathrm{~K}$, the change in entropy is zero (i.e., the association is driven only 

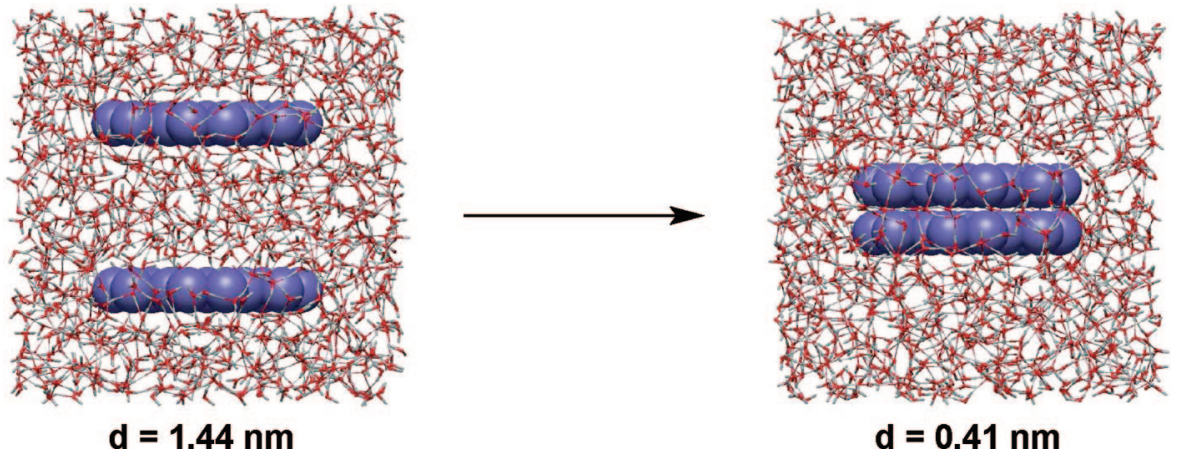

Figure 1. Snapshots of the system: dissociated state $(d=1.44 \mathrm{~nm})$ and associated state $(d=0.41 \mathrm{~nm})$ of hydrophobic plates solvated in water at $T=320 \mathrm{~K}$.

by enthalpy); however, at this point, there is a significant ordering of the interfacial water molecules. The reason that the change in entropy vanishes is due to opposing contributions of orientational and translational entropies of waters near the hydrophobic solute (i.e., they are rotationally ordered and translationally disordered as compared to the bulk). In addition, we studied the temperature dependence of the drying-wetting transition. We found that there was a slight increase of the critical distance for drying as the temperature increased. From calculations of the contact angle of water on the hydrophobic plate at different temperatures, we compared the critical distance observed in the simulations to the critical distance predicted by macroscopic theory. We found that at low temperatures there was an excellent agreement between the predicted and the observed values of the critical distance. As the temperature increased, it appeared as if the agreement became slightly less good; however, it is not clear as to if this is significant.

\section{Materials and Methods}

General Considerations. We studied the effect of temperature on the drying transition as well as on the thermodynamics of the association process of two large hydrophobic surfaces. (Note that the edge surface of these plates is not negligible, and therefore, the system is not purely large-scale. Nevertheless, if one assumes that the edge contribution to the properties of the system is the same when the plates are in contact as when they are separated, then the association process arises only from changes in the large-scale hydrophobic surface. There might still be an effect due to the reduction of curvature of the plate-water interface upon association due to the burial of part of the surface of the atoms at the edge of the plates, although this has yet to be shown.) Each surface is represented by a single-layered plate of 31 atoms arranged in a triangular lattice with a bond length of $0.32 \mathrm{~nm}$. The shape of the plate is disklike with a diameter of $\sim 2.1 \mathrm{~nm}$ (see Figure 1 in a related study ${ }^{28}$ ). The Lennard-Jones (LJ) parameters of the plate atoms are $\sigma_{\text {plt }}=0.40 \mathrm{~nm}$ and $\varepsilon_{\text {plt }}=0.50 \mathrm{~kJ} / \mathrm{mol}$. The two plates were solvated in 1090 water molecules. We chose the SPC/E model ${ }^{49}$ of water and used combination rules (arithmetic average for $\sigma$ and geometric average for $\varepsilon$ ) to calculate the water-plate interactions. Using the Gibbs ensemble Monte Carlo method, the coexisting liquid and vapor densities of SPC/E over a wide range of temperatures were $\operatorname{shown}^{50}$ to be in good agreement with experimental data. Analysis of the water density profile next to the plates ${ }^{28}$ suggests that the strength of the attraction between the water molecules and these plates is very similar to that of water and a hydrocarbon monolayer described at the atomic level. ${ }^{51}$ During simulations, the positions of the plate atoms are held fixed, interactions between atoms on the same plate are excluded, and the orientation of the two plates with respect to each other is parallel and in-registry.

We used the molecular dynamics (MD) package GROMACS version $3.1 .4^{52}$ to perform computer simulations, with a time step of $0.002 \mathrm{ps}$. The bond distances and angles of the water molecules were constrained using the SETTLE algorithm. ${ }^{53}$ The system was maintained at a constant temperature and pressure of 1.0 bar using a Berendsen thermostat. ${ }^{54}$ The electrostatic forces were evaluated by the Particle-Mesh Ewald method (with grid spacing of $0.12 \mathrm{~nm}$ and quadratic interpolation) and the LJ forces by a cutoff of $1.0 \mathrm{~nm}$.

The potential of mean force (PMF) between the two plates was computed from the mean force acting on each of the plates. ${ }^{55,56}$ Then, the mean force acting between the plates along their axis of separation was integrated as a function of the distance between the plates, $d$, to yield the free energy profile. Here, $d$ is defined as the perpendicular distance between the centers of in-registry atoms on different plates so that the interplate distance $D$, defined in the macroscopic theory that gives rise to eq 1 , is $D=d-\sigma_{\text {plt }}$, where $\sigma_{\text {plt }}$ is the diameter of a plate atom. As the PMF represents only relative values, it was shifted such that the free energy of state at the largest separation corresponds to zero. We performed simulations at five temperatures, $T=280,300,320,340$, and $360 \mathrm{~K}$. For each temperature, we performed 54 simulations with different values of plate separation, $d$, ranging from 0.36 to $1.44 \mathrm{~nm}$. At each value of $d$, the system was equilibrated for $2.0 \mathrm{~ns}$, and data were collected for $5.0 \mathrm{~ns}$. At points where the force converged slowly (around the wetting-drying transition), the data collection stage was extended for an additional $5.0 \mathrm{~ns}$. To perform structural and thermodynamic analysis of dissociated, $d=1.44$ $\mathrm{nm}$, and associated, $d=0.41 \mathrm{~nm}$, states (see Figure 1), we performed three additional simulations of $40 \mathrm{~ns}$ for each of these states at each temperature. The error in the quantities obtained from the simulations was estimated using a block averaging method. ${ }^{57}$

Simulations of Water Droplet. To calculate the temperature dependence of the contact angle of a water droplet on the previously mentioned hydrophobic surface, we performed five additional sets of simulations (GROMACS package version 3.3). The structure of the hydrophobic surface was the same as the plates we used for calculating the PMF values (i.e., a triangular lattice with bond length of $0.32 \mathrm{~nm}$ ) with the same LJ parameters for the plate-water interaction. However, in these simulations, the size of the hydrophobic surface was much larger. It was built by 2725 atoms arranged in two layers (out-of-registry with respect to each other) along the $z$-axis and had an area of 121 $\mathrm{nm}^{2}$ in the $x y$-plane. (In principle, the simulations at different temperatures need to take into account the thermal expansion 
of the plates. However, the effect is very small. For example, for graphite, the largest thermal expansion coefficient $\left(\alpha_{\| 1}\right)$ is $6.5 \times 10^{-6} / \mathrm{K}$, which means that a bond length of $0.3200 \mathrm{~nm}$ at $T=280 \mathrm{~K}$ will increase to 0.3202 at $T=360 \mathrm{~K}$.) The water droplet was represented by 1980 SPC/E molecules. The simulations were performed at constant $N V T$, where the size of the simulation box was 11.0, 11.0, and $9.0 \mathrm{~nm}$ along the $x$-, $y$-, and $z$-axis, respectively. In this series of simulations, the temperature was maintained by the Nosé-Hoover thermostat. ${ }^{58,59}$ Initially, a cube of water molecules was placed on the hydrophobic surface. Given the size of the simulation box, a large volume of vacuum surrounded the water molecules. After 400 ps of simulations at $300 \mathrm{~K}$, the water adopted a semispherical shape on the hydrophobic surface. The system was then equilibrated at each temperature $(T=280,300,320,340$, and $360 \mathrm{~K})$ for 2 $\mathrm{ns}$, and data were collected from two independent simulations of $4 \mathrm{~ns}$ each.

The contact angle, $\theta_{\mathrm{c}}$, which is the angle between the liquid-vapor interface and the solid hydrophobic surface, was obtained by determining the liquid-vapor interface of the droplet. ${ }^{60,61}$ The liquid-vapor interface is identified as the location, calculated relative to the center of mass of the droplet, where the density of water falls below $0.5 \mathrm{~g} / \mathrm{cm}^{3}$. Then, the boundary profile of this interface was fit to a circle, excluding data points lower than $0.80 \mathrm{~nm}$ above the surface (due to the oscillatory behavior of the density profile next to the surface). The contact angle between the tangent of the circle and the surface was calculated at a heigh $z=(0.400+0.317) / 2=0.358$ $\mathrm{nm}$ above the center of mass of the outer plate atoms (which is the closest distance SPC/E water molecules can approach the surface).

\section{Results and Discussion}

Thermodynamics of Hydrophobic Association. Figure 2 displays the free energy profile, or PMF, for bringing the two hydrophobic plates from far apart $(d=1.44 \mathrm{~nm})$ to a close distance $(d=0.36 \mathrm{~nm})$ for five different temperatures in the range of $280 \leq T \leq 360 \mathrm{~K}$. The minimum exhibited around $d$ $=0.41 \mathrm{~nm}$ indicates the equilibrium state of the collapsed (associated) state. Therefore, in considering the plate association process (Figure 1)

$$
P(\mathrm{aq})+P(\mathrm{aq}) \Leftrightarrow P_{2}(\mathrm{aq})
$$

we take the state at $d=1.44 \mathrm{~nm}$ as the dissociated state and the state at $d=0.41 \mathrm{~nm}$ as the associated state. The results shown in Figure 2 indicate that the free energy difference, $\Delta G$, between these states (i.e., for the process described in eq 3 ) has a large negative value at all temperatures and that it does not exhibit large variations (at least as compared to its magnitude) for the different temperatures. Looking at Figure 2, we find that $\Delta G$ exhibits a minimum (i.e., a maximum in the strength of hydrophobic interactions) at around $T=340 \mathrm{~K}$. The values of $\Delta G$ for association as a function of the temperature are plotted in Figure 3a. The largest difference between the free energy changes, $\Delta G(T=280 \mathrm{~K})-\Delta G(T=340 \mathrm{~K}) \simeq 8 \mathrm{~kJ} / \mathrm{mol}$, is just larger than the associated error. Thus, we find the change in free energy for plate association to be weakly temperature dependent. Simulations of the hydration of small hydrophobic solutes found an increase in the chemical potential as the temperature increased ${ }^{5,6}$ On the other hand, other simulation studies ${ }^{13,14,62}$ found a maximum (observed at $420 \leq T \leq 470$ $\mathrm{K})$ in the chemical potential of small hydrophobic solutes in water as the temperature increased. It was further found that the maximum is more pronounced and shifts to lower temper-

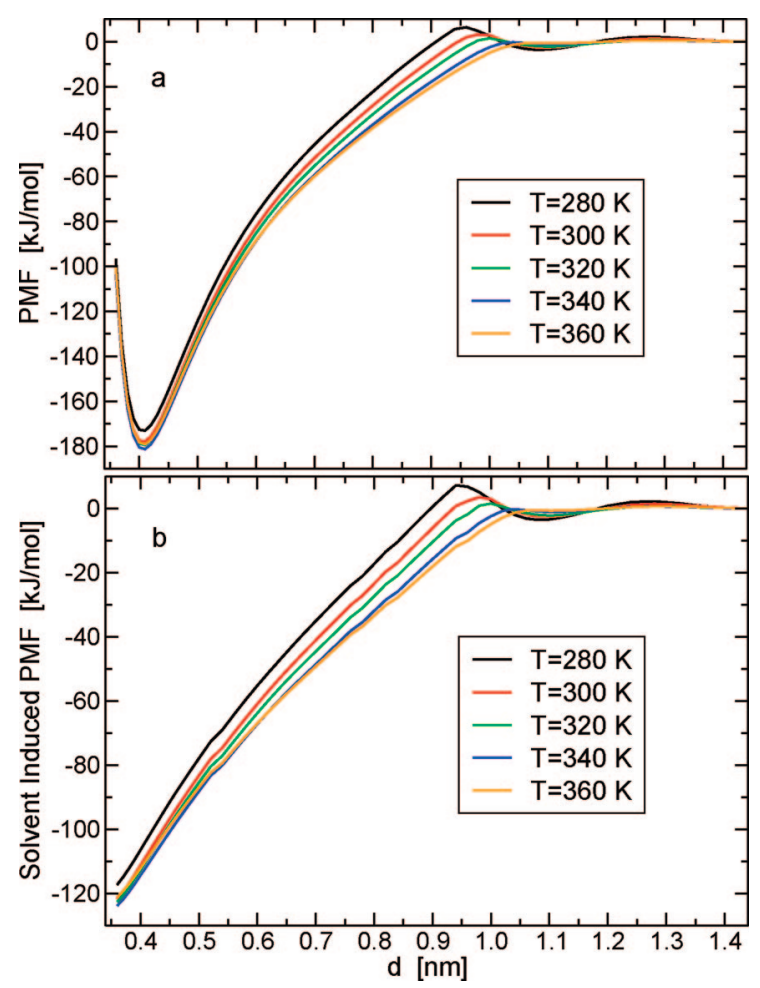

Figure 2. PMF for bringing the two plates from far apart $(d=1.44$ $\mathrm{nm})$ to close contact $(d=0.36 \mathrm{~nm})$ at different temperatures. Total PMF (a) and solvent-induced part of the PMF (b). The estimated error in determining the PMF at contact (relative to the dissociated state) is $3.3 \mathrm{~kJ} / \mathrm{mol}$ for $T=280 \mathrm{~K}$ and decreases to $2.1 \mathrm{~kJ} / \mathrm{mol}$ for $T=360 \mathrm{~K}$.

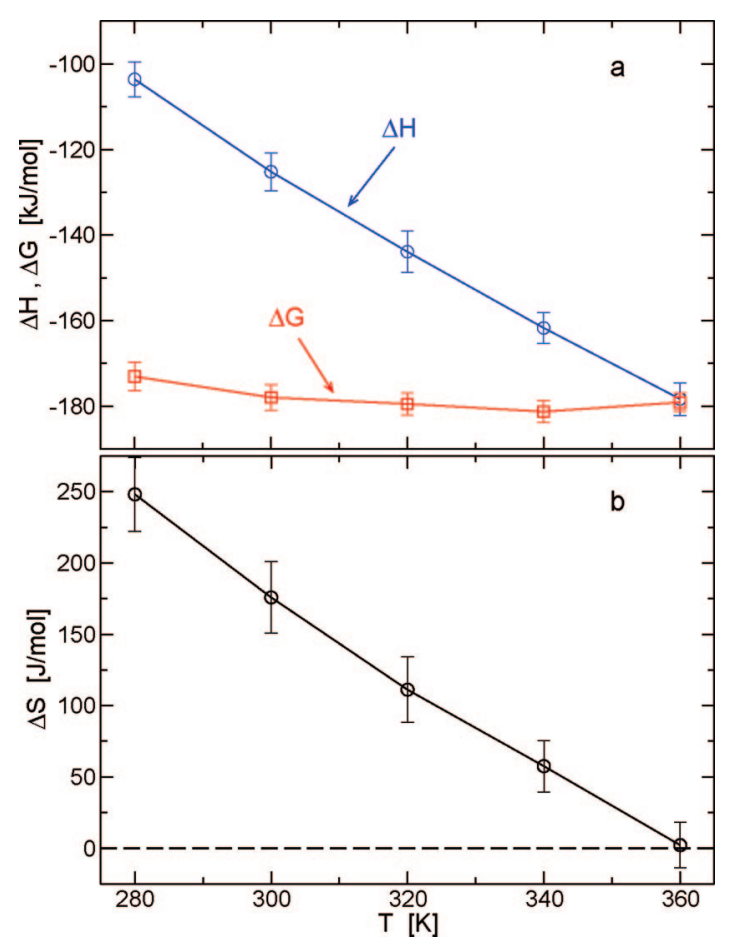

Figure 3. (a) Changes in enthalpy, $\Delta H$, and free energy, $\Delta G$, as well as in (b) entropy, $\Delta S$, for the plate association process (eq 3 ), where the initial state is at $d=1.44 \mathrm{~nm}$ and the final state is at $d=0.41 \mathrm{~nm}$, as a function of temperature.

atures as the size of the solute is increased. Figure $3 \mathrm{a}$ also displays the change in enthalpy (enthalpy change is approximated by the change in energy since the volume change between the two states is negligible), $\Delta H$, for the association 
process of large-scale plates. (To obtain the change in enthalpy that arises only due to the solvent, $\Delta H_{\text {induced, }}$ the direct interaction between the plates at contact, $-69.7 \mathrm{~kJ} / \mathrm{mol}$, has to be subtracted from the values shown in Figure 3a.) This indicates that $\Delta H$ strongly depends on temperature and that the dependence is linear. The value of $\Delta H$ is more negative (larger in magnitude) for higher temperatures. Since the change in heat capacity at constant pressure can be obtained from the slope of this curve

$$
\Delta C_{\mathrm{p}}=\left(\frac{\partial \Delta H}{\partial T}\right)_{\mathrm{p}}
$$

it follows that $\Delta C_{\mathrm{p}}$ is constant in this range of temperatures. This behavior is similar to that observed in the folding of proteins, ${ }^{20,36,39}$ where the folding enthalpy is small at room temperature and becomes large and negative at high temperatures. Another similarity is that the difference in heat capacity between folded and unfolded states is independent of temperature. Nevertheless, $\Delta H$ is shifted toward more negative values for the hydrophobic plates as compared to that for the folding of proteins. This is because in the plates, the exposed hydrophobic region covers a larger area than the exposed area of hydrophobic side chain residues in proteins. From the slope of $\Delta H$ we obtain $\Delta C_{\mathrm{p}}=-930 \mathrm{~J} \mathrm{~mol}^{-1} \mathrm{~K}^{-1}$. The observation that $\Delta G$ does not change much with temperature while $\Delta H$ does implies that there is a compensating contribution from the change in entropy. Figure 3b displays the change in entropy, $\Delta S=(\Delta H-\Delta G) / T$, of the system as a function of temperature. (Note that the change of total entropy in our system is equal to the change of the unitary entropy since the plates do not have any degree of freedom.) There is a linear decrease in the entropy change as the temperature increases. At $T=T_{\mathrm{s}}=360 \mathrm{~K}$, the value of $\Delta S$ is essentially zero. It seems likely that the entropy change for these large-scale hydrophobic plates converges around $T_{\mathrm{s}}$, similar to the behavior observed for the hydration of small hydrophobic solutes and for the folding of proteins. ${ }^{14,19,20}$ At this temperature, the driving force for plate association is only enthalpic. The entropic contribution increases as the temperature decreases. At $T=280 \mathrm{~K}$, the term $T \Delta S=69.4$ $\mathrm{kJ} / \mathrm{mol}$, while the solvent-induced part of the change in enthalpy is $\Delta H_{\text {induced }}=-33.9 \mathrm{~kJ} / \mathrm{mol}$; thus, the solvent-induced driving force for the hydrophobic collapse at low temperatures is more entropic than enthalpic. Note that these results are different from those obtained by Choudhury and Pettitt, ${ }^{63}$ who found the driving force for the association of graphene plates at room temperature to be purely entropic. The reason for the different results is the different strength of the water-plate interaction in the two studies. In our case, the plate-water interactions are weaker than in the study of Choudhury and Pettitt as our aim is to understand hydrophobic association in superhydrophobic plates. With weakening the plate-water interactions, a decrease (and crossover from positive to negative values) of $\Delta H$ is observed. Nevertheless, the temperature dependence will stay the same.

Data $^{36,64}$ for the solvation of hydrophobic compounds (including solids, liquids, and gases as well as for protein denaturation) in water indicate that the ratio between the unitary entropy change and the heat capacity change, $\Delta S_{\mathrm{u}} / \Delta C_{\mathrm{p}}$, has a remarkably constant value of $-0.24 \pm 0.05$. This universal behavior is attributed to the changes in $\Delta S_{\mathrm{u}}$ and $\Delta C_{\mathrm{p}}$ arising from the ordering of the water molecules around the hydrophobic solutes. Later analysis on a larger number of proteins ${ }^{39}$ found this ratio to be spread over a larger range with an average value of $\Delta S$ / $\Delta C_{\mathrm{p}}=-0.126$. In our case (sign of $\Delta S / \Delta C_{\mathrm{p}}$ is the same for
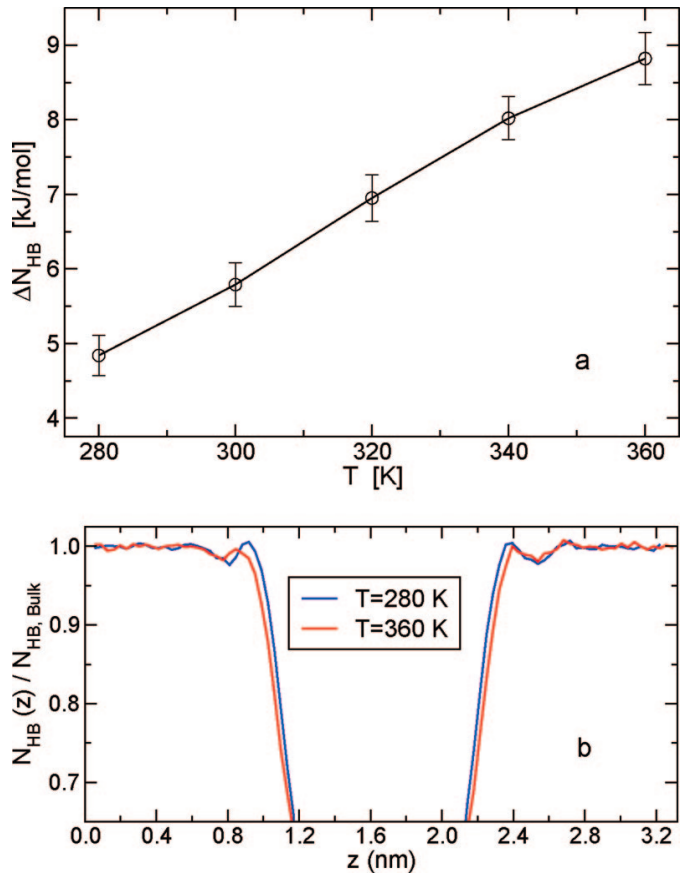

Figure 4. (a) Difference in total number of hydrogen bonds in the system for the association process (i.e., it gives the number of hydrogen bonds formed between water molecules during the association process) as a function of temperature. (b) Number of hydrogen bonds along the $z$-axis normalized by the number of hydrogen bonds in the bulk for $T$ $=280$ and $360 \mathrm{~K}$ for a configuration when the plates are in contact $(d$ $=0.41 \mathrm{~nm}$ ) and centered at $z=1.650 \mathrm{~nm}$. The graph shows that upon heating, the number of hydrogen bonds next to the plates decreases more than in the bulk.

association and dissociation processes), the ratio $\Delta S / \Delta C_{\mathrm{p}}$ is $-0.19 \pm 0.06$.

The value of $\Delta H$ for hydrophobic association is negative because at the surface between plate and water, the water molecules cannot maintain the same number of hydrogen bonds as they can in the bulk. Thus, in the associated state of the plates, the number of hydrogen bonds between the water molecules is larger than in the dissociated state (i.e., there are less broken hydrogen bonds in the associated state). Why, then, does this negative value of $\Delta H$ for hydrophobic association increase in magnitude with temperature? Figure 4a displays the change in total number of hydrogen bonds (hydrogen bond was defined by a donor-acceptor cutoff distance of $0.35 \mathrm{~nm}$ and a donor-hydrogen-acceptor cutoff angle of $30^{\circ}$ ) of water, $\Delta N_{\mathrm{HB}}$, as a function of temperature. As the temperature increases, $\Delta N_{\mathrm{HB}}$ increases as well. Because hydrogen bonds at the surface are more fragile, more hydrogen bonds (per water molecule) break at the surface as compared to the bulk when the temperature is increased. This is shown in Figure $4 b$ where the number of hydrogen bonds normalized by the number of hydrogen bonds in the bulk, as a function of the $z$-axis, is plotted for $T=280$ and $360 \mathrm{~K}$. In this analysis, the configuration of the plate is at contact $d=0.41 \mathrm{~nm}$ and centered at $z=1.650 \mathrm{~nm}$. Only water molecules that are found inside a cylinder with a radius (in the $x y$-plane) of $0.80 \mathrm{~nm}$ around the hydrophobic plates are considred. It is apparent that at high temperatures $(T=360 \mathrm{~K})$, the number of hydrogen bonds at the interface, relative to the bulk, is smaller than at low temperature $(T=280 \mathrm{~K})$.

Why do more hydrogen bonds at the interface break with increasing temperature than in the bulk? One possible explanation is that at the interface, the strength of the hydrogen bonds is weaker than in the bulk. However, since hydrogen bonds are, 


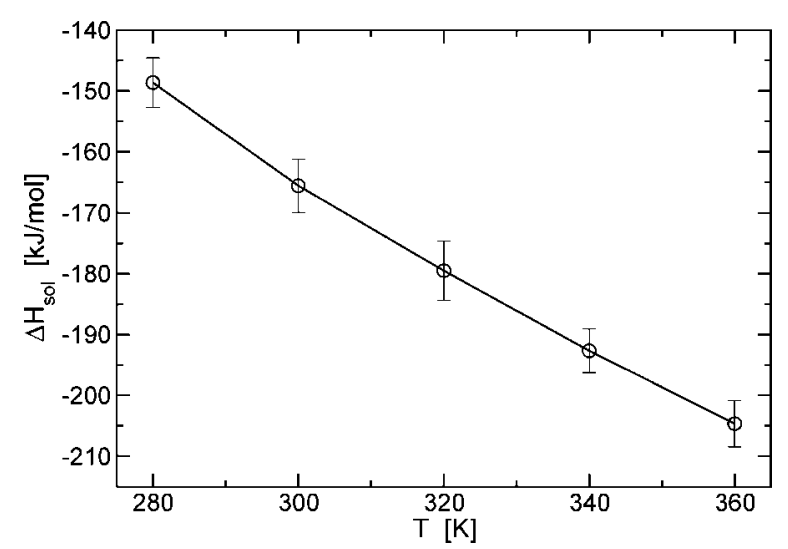

Figure 5. Solvent (water) reorganization enthalpy change, $\Delta H_{\mathrm{sol}}$, for the association process as a function of temperature. Comparison of the slope of this curve to that of $\Delta H$ (shown in Figure 3a) indicates that the major contribution $(\sim 75 \%)$ for the large negative heat capacity change of hydrophobic association arises from changes in the reorganization energy between the water molecules. The results further indicate that as the temperature increases, the coalescence of (largescale) cavities is increasingly favored by enthalpy.

to a large extent, electrostatic in nature, they should be stronger in lower dielectric medium, thus at the interface with the hydrophobic surface. In our opinion, the only way hydrogen bonds at the interface can be weaker than in the bulk is due to larger distortions in the donor-acceptor angle in the former, which is a result of the incompatibility of a planar surface with the tetrahedral network. In addition, we propose another explanation as to why more hydrogen bonds at the interface break with an increase in temperature. The argument is as follows. With increasing temperature, the rotational mobility of the (water) molecules increases. In the bulk, there is a degeneracy in the arrangement of the hydrogen bonds between the water molecules (giving rise to the large residual entropy of ice ${ }^{65}$ ). Therefore, if the rotations of neighboring molecules are correlated, at high temperatures, the water molecules can rotate and at the same time maintain a high degree of tetrahedral network connectivity. However, at the interface, the water molecules adopt a preferential orientation that limits the degeneracy of arranging their hydrogen bonds. As a consequence, with an increase of temperature, their ability to rotate and at the same time maintian hydrogen bond connectivity decreases. It would be very interesting to investigate this explanation further.

The fragility of the interfacial hydrogen bonds is the reason for the large heat capacity in hydrophobe solvation. In Figure 5 we plotted the solvent reorganization change in enthalpy, $\Delta H_{\text {sol }}$ (i.e., it was obtained by subtracting the enthalpy change for the plate-plate and water-plate interactions from the total enthalpy change). The slope of this curve is $700 \mathrm{~J} \mathrm{~mol}^{-1} \mathrm{~K}^{-1}$, which gives the contribution of solvent reorganization to the heat capacity change. Thus, the major contribution $(\sim 75 \%)$ of the large heat capacity change arises from the change of reorganization energy between water molecules around the hydrophobic plates. This is in agreement with many theories of the hydrophobic effect (see, i.e., a recent review on this subject ${ }^{33}$ ). Note that Figure 5 also indicates that there is a larger enthalpic driving force for large-scale cavity coalescence at higher temperatures.

For a process with a large change in heat capacity, the change in entropy is a strong function of temperature, and this is observed in Figure $3 \mathrm{~b}$. Figure $3 \mathrm{~b}$ also indicates that as the temperature increases, $\Delta S$ decreases and is almost zero at $T=$
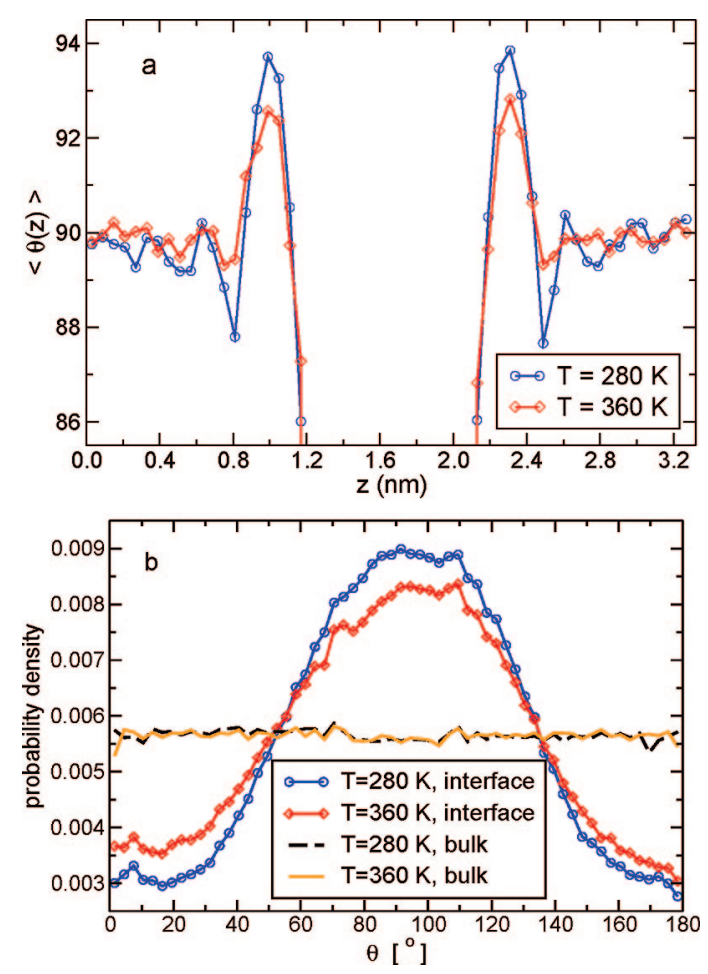

Figure 6. Angle, $\theta$, between the dipole moment vector of the water molecules (direction of the vector is from the positive to the negative charge) and the normal to the plates for a configuration when the plates are in contact $(d=0.41 \mathrm{~nm})$ for $T=280$ and $360 \mathrm{~K}$. (a) Av angle along the $z$-axis. Values larger than $90^{\circ}$ indicate a preference for water oxygen to reside next to the hydrophobic plates. (b) Probability density of $\theta$ for the interfacial layer $(0.80 \leq z \leq 2.50 \mathrm{~nm})$ and for the bulk region $(0.55 \geq z \geq 2.75 \mathrm{~nm})$. It was obtained from the distribution of $\theta$ normalized by the cone angle $\sin \theta$.

$360 \mathrm{~K}$. What is the reason for this behavior? Since the plates are frozen during simulations, $\Delta S$ of the system represents the change of unitary entropy (i.e., the change in entropy of the water molecules). The values of $\Delta S$ are positive for the plate association process (at least for $T<360 \mathrm{~K}$ ). This is in agreement with a model in which water molecules next to hydrophobes are more constrained and ordered than in the bulk. It might be thought that the entropy change is reduced with an increase in temperature due to a decrease in water ordering at the interface. If this is true, then at $T=360 \mathrm{~K}$, the structure of the interfacial water and bulk water would seem to be the same since $\Delta S$ vanishes. However, we will show below that this is not the case.

The preferential orientation of water molecules along the $z$-axis at $T=280$ and $360 \mathrm{~K}$ is shown in Figure 6. We calculated the angle, $\theta$, between the dipole moment vector of the water molecule (the direction is from the positive to the negative end) and the surface outer normal. For a random angular distribution, $\langle\theta\rangle=90^{\circ}$. In Figure $6 a$, the value of $\langle\theta\rangle$ along the $z$-axis is plotted (with similar settings described for Figure $4 \mathrm{~b}$ ). For distances far away from the plates, $\langle\theta\rangle=90^{\circ}$ at both temperatures as expected for the random distribution in bulk water. At the interface with the hydrophobic plates, $\langle\theta\rangle$ is larger than $90^{\circ}$. It is $\sim 94$ and $93^{\circ}$ for $T=280$ and $360 \mathrm{~K}$, respectively. This indicates a preference for the dipole moment to point away from the bulk, similar to the orientation of water molecules next to hydrophobic surfaces found in earlier studies. ${ }^{66-68}$ The probability density of $\theta$ (i.e., the distribution of $\theta$ normalized by the cone angle $\sin \theta)$ in the first layer $(0.80 \leq z \leq 2.50 \mathrm{~nm})$ and in the bulk $(0.55 \geq z \geq 2.75 \mathrm{~nm})$ is shown in Figure 6b. A uniform distribution is displayed for the bulk at both temper- 

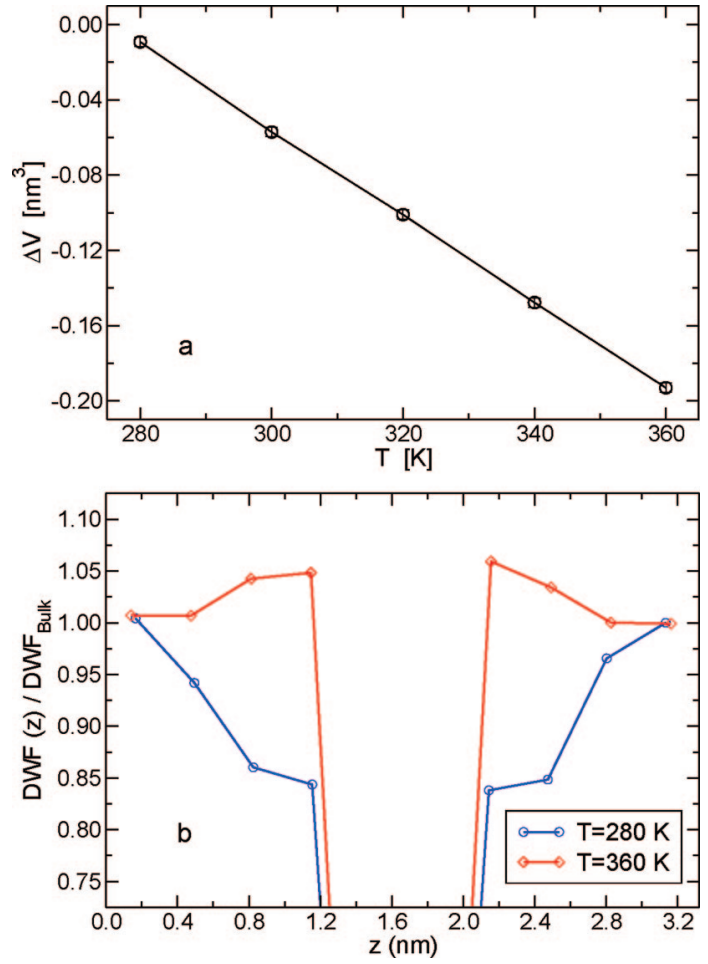

Figure 7. (a) Change in volume of the system for the association process as a function of temperature. The graph shows that the thermal expansion coefficient of interfacial waters is larger than bulk waters. (b) Value of Debye-Waller factor (as defined by eq 6) along the $z$-axis normalized by its bulk value for $T=280$ and $360 \mathrm{~K}$.

atures, while at the interface there is a Gaussian-shaped curved centered around $100^{\circ}$, with a larger magnitute for $T=280 \mathrm{~K}$. This means that at lower temperatures, there is a larger degree of preferential orientation. However, Figure 6a,b clearly indicates that there is a substantial ordering of interfacial water molecules even at $T=360 \mathrm{~K}$ where the change in entropy vanishes (Figure 3b). In fact, the loss of ordering at the interface due to heating is rather small and by itself cannot explain the decrease of $\Delta S$ with temperature.

Another property that can explain the behavior of $\Delta S$ is the change in the density of interfacial water as compared to bulk water. In Figure 7 a we plotted the volume change (of the whole system), $\Delta V$, that occurs for the association process as a function of temperature. The value of $\Delta V$ decreases linearly with temperature. This decrease indicates that the thermal expansion coefficient of water at the interface is larger than that in the bulk (since the net effect of the association process is a reduction of hydrophobic surface area). The same behavior was reported experimentally $^{69}$ by measuring the partial molal volume of hydrocarbons in water at temperatures ranging from 10 to 40 ${ }^{\circ} \mathrm{C}$. Thus, at high temperatures, there is an increase in volume per water molecule at the interface as compared to the bulk. This is in agreement with the previous observation that at high temperatures, more hydrogen bonds are broken at the interface as compared to the bulk. The observed decrease in the density of the interfacial water molecules can increase the translational entropy of the waters at the interface relative to that in the bulk.

To characterize the magnitude of the $z$-component of the translational displacement of water molecules as a function of their position along the $z$-axis, we calculated the local Debye-Waller factor for particle $i$ at a short amount of time by ${ }^{70}$

$$
\mathrm{DW}_{i}=\left\langle\left\langle\left(\vec{z}_{i}(t)-\left(\vec{z}_{i}\right)_{\tau}\right)^{2}\right\rangle_{\tau}\right\rangle
$$

where $\langle\ldots\rangle_{\tau}$ denotes the average over the time period $\tau$ and the outer brackets denote the average over the simulation trajectory (i.e., average over different time origins). The value of the $z$-coordinate of each water oxygen, calculated at the beginning of each time interval $\tau$, was used to assign the water molecules to slices along the $z$-axis

$$
\mathrm{DW}(z)=\left\langle\mathrm{DW}_{i} \delta\left(z_{i}-z\right)\right\rangle
$$

However, if at any time during $\tau$ a water molecule exits the initially assigned slice, the displacement of this particle during this time interval was not included in the average in eq $6 .^{71}$ The function $\operatorname{DW}(z)$ for $\tau=150$ ps divided by its bulk value is displayed in Figure $7 \mathrm{~b}$ for $T=280$ and $360 \mathrm{~K}$ (with similar settings described for Figures $4 \mathrm{~b}$ and $6 \mathrm{a}$ ). It shows that at low temperatures $(T=280 \mathrm{~K})$ next to the hydrophobic plates, the mobility of the water molecules is retarded as compared to the bulk, while at high temperatures $(T=360 \mathrm{~K})$, the mobility is enhanced relative to the bulk. This means that at low temperatures, interfacial waters have a lower, while at high temperatures they have a higher, translational entropy as compared to bulk water. This is expected from the behavior of $\Delta V$ and is consistent with the reduction of $\Delta S$ as the temperature increases. At $T=T_{\mathrm{s}}$, the higher translational entropy exactly compensates for the lower orientational entropy of interfacial waters relative to bulk waters, revealing the physical picture at the point of zero entropy $\left(\Delta S\left(T=T_{\mathrm{s}}\right)=0\right)$. Therefore, the assumption that at this temperature there is no ordering of the water molecules at the interface is not correct; there is orientational order and translational disorder of the water molecules at the interface relative to the bulk. Note that the hindered motion we find here at low temperatures resembles that of water molecules next to hydrophobic regions of protein surfaces at ambient conditions. ${ }^{72}$

Drying Transition and Contact Angle of Water. Analysis of the simulation trajectories indicates that the strength of the water-plate interaction (i.e., the LJ parameters and geometry of the plate atoms) constitutes a hydrophobic surface that exhibits drying transition at all temperatures studied. Figure 8a exhibits a snapshot of the simulation box at $T=320 \mathrm{~K}$. The distance between the plates is $d=1.00 \mathrm{~nm}$. Only a slice along the $x z$-plane (the plane of the paper) with a thickness of 1.40 $\mathrm{nm}$ along the $y$-axis is shown. It is clear that for this interplate distance, the water molecules are repelled from the region between the two plates. Since the diameter of the plate atoms is $0.40 \mathrm{~nm}$, the space between the plates has a thickness of 0.60 $\mathrm{nm}$, which can be occupied by at least two layers of water molecules. Bringing the plate from far apart, where the region between the plate is filled with water (the wet state), to a distance just smaller than the critical distance involves cavitation, the formation of a bubble. The reversible work associated with bringing the plates from the wet state to a distance that allows the onset of the dry state can be obtained from the PMF shown in Figure 2 as the difference between the free energy of the maximum at the critical distance $d=0.95-1.05 \mathrm{~nm}$ and the adjacent minimum at a larger distance. This free energy barrier, $\Delta G^{\ddagger}$, as a function of temperature is plotted in Figure $8 \mathrm{~b}$. The value of $\Delta G^{\ddagger}$ decreases as the temperature increases, for example, at $T=280 \mathrm{~K}, \Delta G^{\ddagger}=10.0 \mathrm{~kJ} / \mathrm{mol}$ while at $T=360$ $\mathrm{K}$, it is $0.1 \mathrm{~kJ} / \mathrm{mol}$. The decrease of $\Delta G^{\ddagger}$ with increasing temperature is expected because the difference in the chemical potential of the vapor and the liquid states decreases as the temperature is increased (up to the boiling temperature). Note that at the critical distance the free energy of the wet and dry states is the same and separated by a barrier. The system, then, 

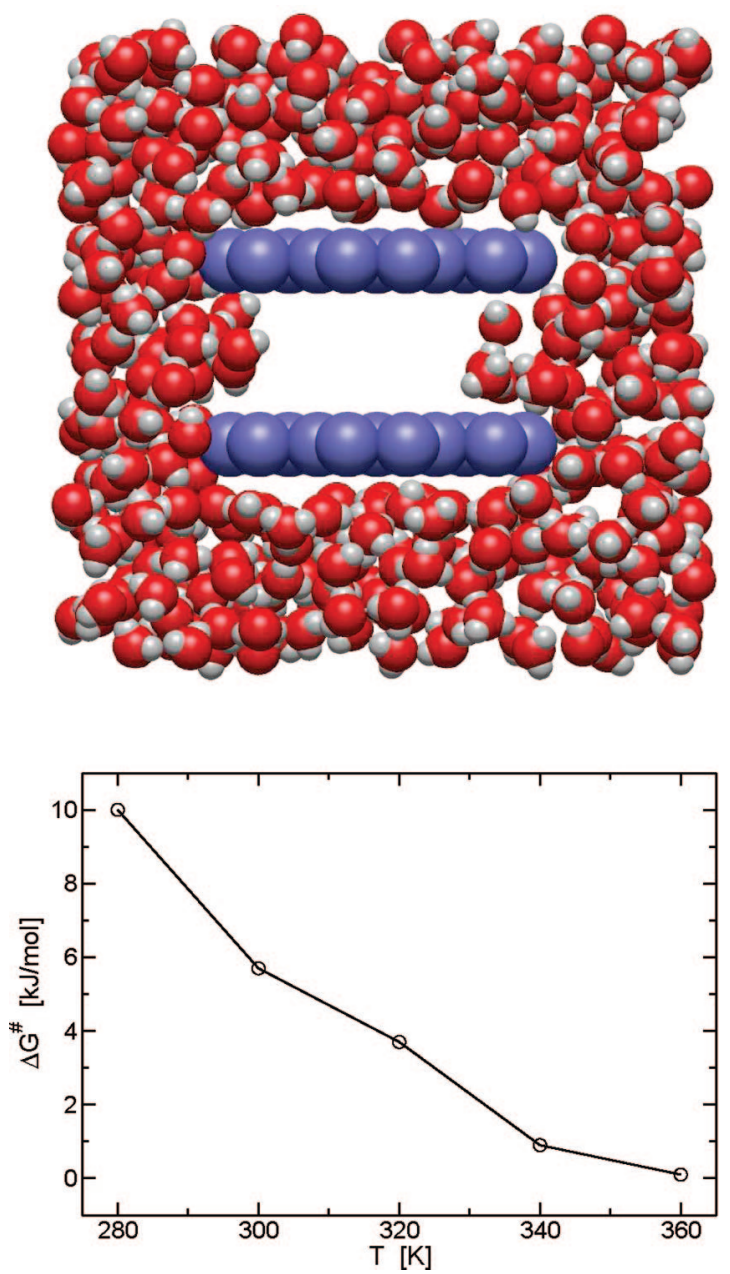

Figure 8. Top panel: snapshot from the simulation at $T=320 \mathrm{~K}$ showing the drying transition. For clarity, only a slab along the $x z$ plane (plane of the paper) with a thickness of $1.40 \mathrm{~nm}$ around the plates is shown. The distance between the two hydrophobic plates is 1.00 $\mathrm{nm}$. The diameter of the plate atoms is $0.40 \mathrm{~nm}$; thus, the free space between the plates has a thickness of $0.60 \mathrm{~nm}$ that can accommodate at least two layers of water. Lower panel: reversible work (calculated from the PMF shown in Figure 2) for cavitation (bubble formation) as a function of temperature.

fluctuates between these states with a kinetics that is determined by the height of this barrier, which is different than the value of $\Delta G^{\ddagger}$ plotted in Figure $8 \mathrm{~b}$.

To determine the critical distance for the drying transition, we calculated the number of water molecules, $N_{\text {water }}$, confined between the two surfaces inside a cylindrical volume with a radius of $0.80 \mathrm{~nm}$ in the $x y$-plane as a function of distance between the plates, $d$. The curves of $N_{\text {water }}(d)$ for $280 \leq T \leq$ $360 \mathrm{~K}$ are displayed in Figure 9a. At all temperatures, the curve exhibits a sharp decrease of $N_{\text {water }}$ in the range of $0.95 \lessgtr d \lesssim$ $1.05 \mathrm{~nm}$ when the plates are brought into contact. (Note that at lower temperatures $(T=280 \mathrm{~K})$, the transition is sharper than at higher temperatures $(T=360 \mathrm{~K})$.) This signifies the distance between the plates at which the drying transition takes place, $D_{\text {c }}$. The value of $D_{\text {c }}$ (presented as the distance available for the water molecules confined between the two plates and obtained by subtracting the diameter of the plate atoms, $0.40 \mathrm{~nm}$, from the values inferred from Figure 9a) as a function of temperature is plotted in Figure 9b. The gray area marks the uncertainty in determining $D_{\mathrm{c}}$ (distance at halfway from transition borders). It is specified by the boundaries of the sharp decrease in $N_{\text {water }}(d)$. The curve shown in Figure 9b exhibits a slight increase
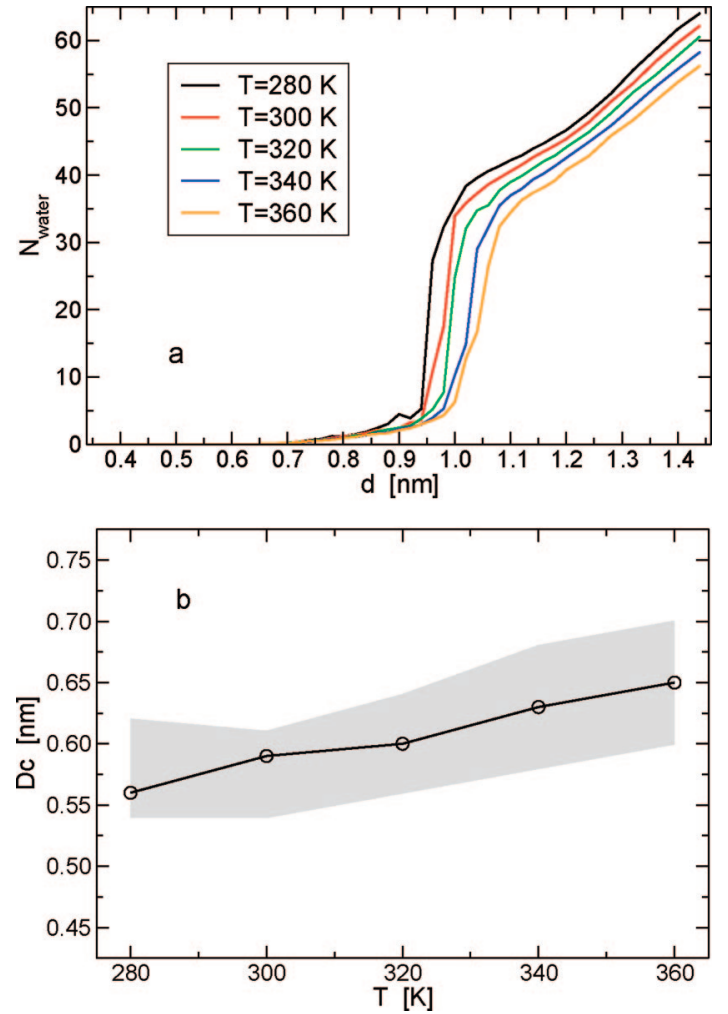

Figure 9. Drying transition: (a) number of water molecules between plates (inside a cylinder with a radius of $0.8 \mathrm{~nm}$ ) as a function of interplate distance for different temperatures. (b) Critical distance, $D_{\mathrm{c}}$, for the drying transition as a function of temperature. The gray area marks the uncertainty in determining the critical distance. Note that the distances, $d$, in panel a were calculated from the center of mass of the plate atoms; however, the critical distance shown in panel $b$ is the distance, $D$, available for the water molecules confined between the plates and is calculated by subtracting the diameter of the plate atoms from the values inferred from panel a; thus, $D=d-\sigma_{\text {plt }}$.

in the critical distance with temperature, implying that, effectively, the plates become more hydrophobic with increasing temperature (within the temperature range studied). (In principle, hysteresis, characterizing first-order transitions, can be observed in Figure 9a. However, since the system is finite, the transition is less abrupt than a first-order transition in the thermodynamic limit, and we did observe multiple transitions (four at $T=280$ $\mathrm{K}$ and 20 at $T=360 \mathrm{~K}$ ) around $D_{\mathrm{c}}$, between empty and filled states. Although the estimated error in determining the number of water molecules between the plates is relatively large around $D_{\text {c }}$, we do not expect a large hysteresis.)

As mentioned in the Introduction, a macroscopic thermodynamic analysis relates the critical distance for drying between two hydrophobic plates, $D_{\mathrm{c}}$, to the contact angle $\theta_{\mathrm{c}}$ of a water droplet on the hydrophobic plate, $D_{\mathrm{c}} \simeq-R_{\mathrm{m}} \cos \theta_{\mathrm{c}}$. We are interested in testing the validity of this macroscopic theory at different temperatures. Thus, an independent determination of $\theta_{\mathrm{c}}$ at different temperatures is required. To this end, we conducted additional simulations of a water droplet on a large hydrophobic surface at different temperatures (see Materials and Methods). Figure 10 displays side and top view snapshots from these simulations at $T=280,320$, and $360 \mathrm{~K}$. Note that at $T=$ $360 \mathrm{~K}$, few of the water molecules are evaporated. A schematic representation of the procedure for calculating the contact angle of water on the hydrophobic surface is shown in the lower panel of Figure 10. The empty black circles are data points obtained from simulations for the liquid-vapor interface of the water droplet while the circular red line is a fit to these data points. 

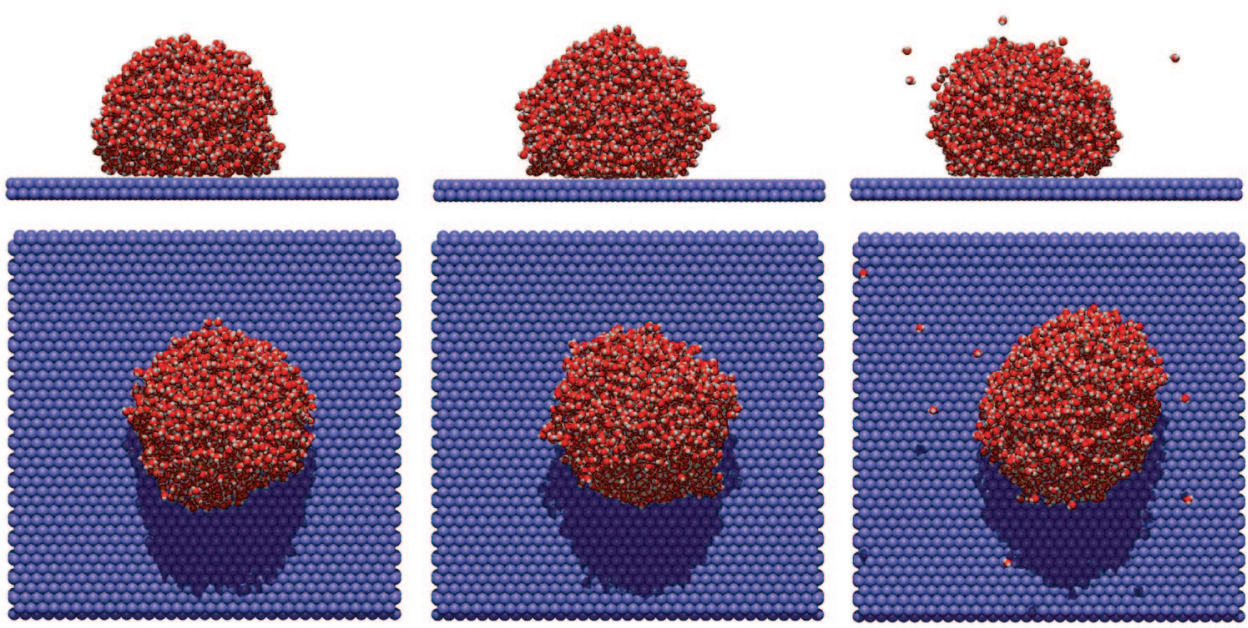

$\mathrm{T}=280 \mathrm{~K}$

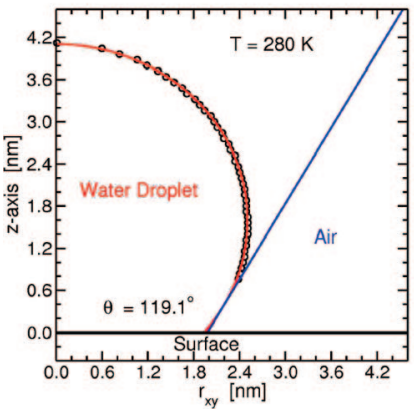

$\mathrm{T}=320 \mathrm{~K}$

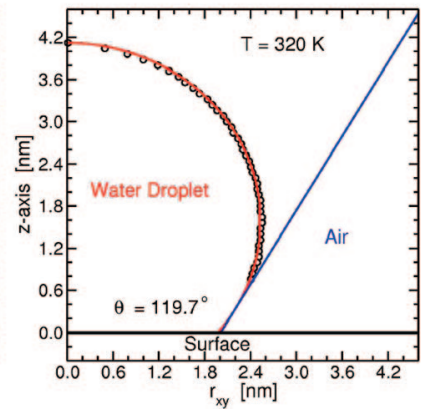

$\mathrm{T}=360 \mathrm{~K}$

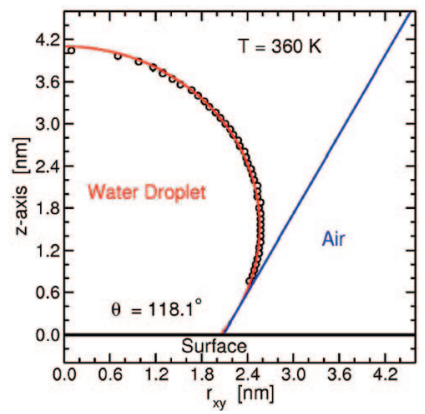

Figure 10. Top and middle panels: side and top view snapshots, respectively, of a water droplet on a hydrophobic surface. Lower panel: radial boundary (lateral component from the drop center of mass) of the water-air interface along the $z$-axis (surface normal) of the droplet. The empty black circles are data points from the simulations, while the red circular solid line is a fit to these points. The contact angle at $z=0.358 \mathrm{~nm}$ above the center of mass of the plate atoms is also shown. Left, middle, and right columns correspond to $T=280,320$, and $360 \mathrm{~K}$, respectively.

Utilizing the modified Young's equation, ${ }^{60}$ the microscopic contact angle obtained from the simulations, $\theta_{0}$, is related to the macroscopic contact angle, $\theta_{\infty}$, through

$$
\cos \theta_{\infty}=\cos \theta_{0}+\frac{\tau}{\gamma_{1 \mathrm{v}}} \frac{1}{r_{\mathrm{B}}}
$$

where $\tau$ is the line tension and $r_{\mathrm{B}}$ is the droplet base radius. To calculate $\theta_{\infty}$ at different temperatures, we need values of $\gamma_{\mathrm{lv}}, \tau$, and $r_{\mathrm{B}}$ at these temperatures. The value $r_{\mathrm{B}}$ can be calculated from the simulations and was found to be in the range of 2.51 and $2.56 \mathrm{~nm}$ for $T=280$ and $360 \mathrm{~K}$, respectively. The values of the liquid-vapor surface tension of water as a function of temperature were taken from Schonhorn. ${ }^{73}$ Note that these experimental values can differ from those of SPC/E water and, therefore, can contribute an error in determining $\theta_{\infty}$. Furthermore, to obtain the values of $\tau$ as a function of temperature, additional sets of simulations are needed. Specifically, at each temperature, $\tau$ can be calculated from the dependency of the contact angle on the size of the droplet. However, since this would require additional intensive computations, we decided to take an approximate value from the literature. Simulation studies of (SPC/E) water on a graphite surface ${ }^{60}$ found the line tension to be in the range of $0.7-2.7 \times 10^{-10} \mathrm{~J} / \mathrm{m}$ depending on the strength of the carbon-water interaction. Interpolating the strength of the surface water interaction used in our study to simulations carried out by Werder et al. ${ }^{60}$ suggest a value of $\tau \approx 2.5 \times 10^{-10} \mathrm{~J} / \mathrm{m}$. We assumed this value for all temperatures studied. The values of $\theta_{0}$ and $\theta_{\infty}$ as a function of temperature are plotted in Figure $11 \mathrm{a}$, indicating that the difference between $\theta_{0}$ and $\theta_{\infty}$ is in the range of $1.3-1.6^{\circ}$. The values shown are averaged over two independent simulations, where the extrema of the error bars mark the values obtained from each simulation. There is a very slight decrease in the contact angle as a function of temperature, and it is not clear as to if this decrease is significant.

Experimentally, there are only a few reports on the dependency of contact angles on temperature. It has been argued that the corresponding temperature coefficient is very small if not zero. ${ }^{74}$ For example, the average coefficient measured for water on polyethylene ${ }^{75}$ is $-0.045^{\circ} / \mathrm{K}$, on polystyrene ${ }^{76}$ it is $-0.025^{\circ}$ / $\mathrm{K}$, and on naphthalene ${ }^{76}$ it is $-0.13^{\circ} / \mathrm{K}$. For a temperature range of $80 \mathrm{~K}$, that amounts to a decrease in contact angle by 3.6, 2.0 , and $10.4^{\circ}$, respectively. Furthermore, the contact angle of water on silicone rubber ${ }^{77}$ in the temperature range of $273 \leq T$ $\leq 353 \mathrm{~K}$ increased by $4^{\circ}$ and remained essentially unchanged on silicone glass. ${ }^{74}$ The insensitivity of the contact angle to temperature is reported to hold in other systems as well. ${ }^{73}$ An argument that explains this weak dependence of contact angle on temperature is as follows: ${ }^{78}$ Young's equation can be written as

$$
W_{\mathrm{sl}}=\gamma_{\mathrm{lv}}\left(1+\cos \theta_{\mathrm{c}}\right)
$$

Thus, two factors affect the contact angle: the surface tension of the liquid-vapor interface, $\gamma_{\mathrm{lv}}$, and work of adhesion, $W_{\mathrm{sl}}$, 

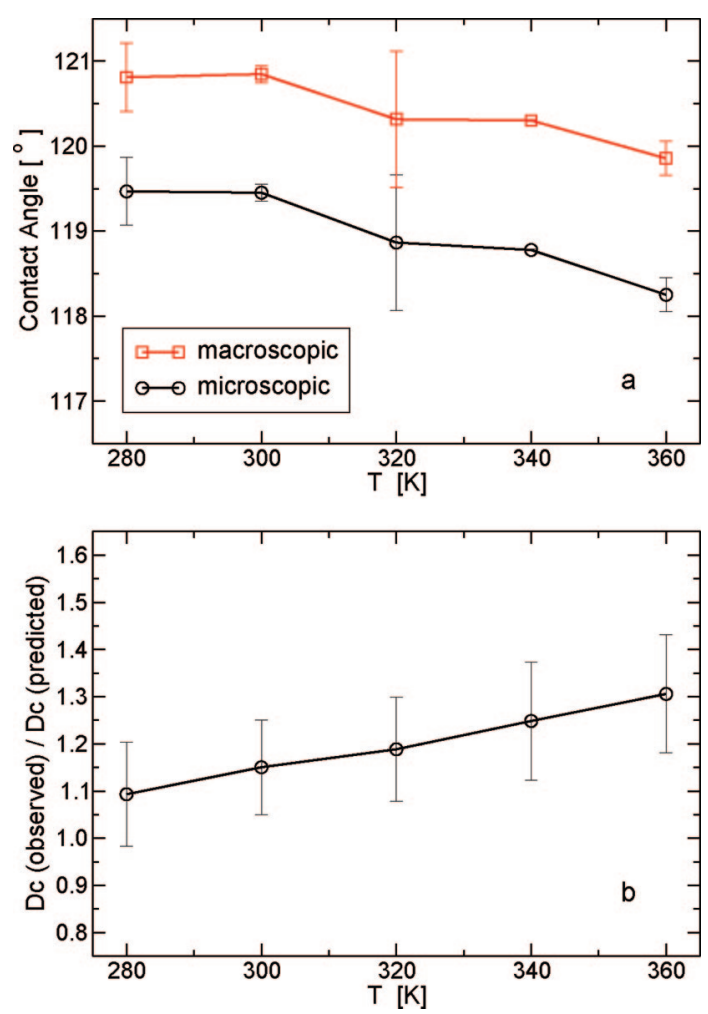

Figure 11. (a) Microscopic contact angle, $\theta_{0}$ (obtained directly from simulation of the droplet), and macroscopic contact angle, $\theta_{\infty}$ (corrected to infinitely large droplet size), of water on the hydrophobic plate. The values shown were averaged over two simulations where the width of the error bars indicates the difference between the two simulations. (b) Critical distance observed in the simulations (from Figure 9) divided by the critical distance predicted by macroscopic theory based on the value of the macroscopic contact angle shown in panel a.

the work required to separate the liquid from the solid. It has been suggested that the temperature change of liquid-vapor surface tension is balanced by the temperature change of the adhesive force between the liquid and the surface. ${ }^{78}$

The values of the contact angles we obtained from this set of simulations allow us to use macroscopic theory to predict a critical distance for the drying transition, $D_{\mathrm{c}}=-R_{\mathrm{m}} \cos \theta_{\infty}$, where $R_{\mathrm{m}}=1.0 \mathrm{~nm}$, the radius of the disk-like plates. Since a realistic estimation for the error in determining the contact angle is not possible based on the two simulations we performed at each temperature, we take the difference between $\theta_{0}$ and $\theta_{\infty}$ as an estimation for this error. The values of the predicted critical distance decreased from 0.512 to $0.498 \mathrm{~nm}$ with increasing the temperature from $T=280$ to $360 \mathrm{~K}$. The observed values of $D_{\mathrm{c}}$ from the simulation increased from 0.56 to $0.65 \mathrm{~nm}$ as the temperature increased within this temperature range. It is remarkable that a simple theory, based on thermodynamic considerations of macroscopic properties, predicts to good accuracy (relative error of $8-23 \%$ ) a phenomenon that occurs at the molecular level. Figure 11b displays the ratio between observed and predicted values of $D_{\mathrm{c}}$ as a function of temperature. Although the deviations of the curve from 1.0 are relatively small, there seems to be a systematic increase in the ratio as the temperature increases. As discussed previously, we do expect an error due to the fact that in the calculation of $\theta_{\infty}$ we took the value of $\tau$ to be the same for all temperatures. It is likely that this value of the line tension will change with temperature. If $\tau$ increases with temperature, then the curve in Figure 11b will be more horizontal, while if $\tau$ decreases with temperature, the curve will have a larger slope exhibiting larger deviations at high temperatures. In the case that the latter holds, what would be the reason as to why the macroscopic theory applies better at low temperatures than at high temperatures? In a thermodynamics framework, it is assumed that the distribution of thermodynamic functions sharply peaked around their average values and that any deviation from the average is ignored. This description ignores fluctuations in the system. However, since the relative fluctuations in the system reduce with system size, the thermodynamics relations are exact when the number of degrees of freedom approaches infinity. In contrast, the drying transition studied in our simulations is better represented by thermodynamics of finite systems. ${ }^{79}$ From analysis of the transition states for the drying transition (results are not shown), we find that, although the average number of water molecules confined between the two plates is between the filled state and the empty state, the distribution of this number is bimodal. That means that for some time, the region between the plates is filled with water, while for other times it is empty. This describes large fluctuations in the system that characterize finite systems. The extent of these fluctuations increases with increasing temperature, and they might offer an explanation for the slight increase in the deviation between observed and predicted values of $D_{\mathrm{c}}$. The observation that the transition is sharper at lower temperatures than at higher temperatures (see Figure 9a) is consistent with the fact that at lower temperatures, the system is better described in a thermodynamic limit of a first-order transition.

\section{Conclusion}

The behavior of phenomena associated with hydrophobic interactions can be qualitatively different depending on as to whether the interacting hydrophobic regions are small or large. In this study, we investigated the temperature behavior of largescale hydrophobic association and found characteristics similar to the observed temperature behavior of small hydrophobic solutes. Specifically, the changes in enthalpy and entropy for large-scale hydrophobic association are strong functions of temperature, while the change in free energy is very weakly temperature dependent. In addition, we found that at $T=T_{\mathrm{s}}=$ $360 \mathrm{~K}$, the change in entropy vanishes, and different large-scale hydrophobic solutes are likely to exhibit entropy convergence at this temperature. Modeling extended hydrophobic regions allowed us to elucidate the molecular mechanism that explains the zero entropy change at $T_{\mathrm{s}}$. In contrast to suggestions that at $T_{\mathrm{s}}$ the interfacial water molecules are structurally indistinguishable from bulk waters, we find that at this temperature the water molecules around hydrophobic solutes are orientationally ordered and translationally disordered relative to bulk water. These opposing contributions to the entropy change cancel each other exactly at $T_{\mathrm{s}}$. This kind of cancelation has not yet been shown for small-scale hydrophobic association. The findings we obtained here are important for interpretation of the temperature behavior and thermodynamics of protein folding. In addition, we investigated the temperature dependence of the drying-wetting transition. Specifically, we tested the validity of a macroscopic thermodynamic theory for predicting the critical distance at different temperatures. We found that the macroscopic theory predicts remarkably well the drying transition, which is a microscopic phenomenon. The deviations of the predicted, from the observed, values of the critical distance are very small; nevertheless, the agreement seems to be better at lower temperatures. It is not clear as to whether these deviations are significant; however, we propose that it might be a result of stronger fluctuations (which are ignored in the usual thermo- 
dynamic description) at higher temperatures that cannot be neglected in the thermodynamics of small systems.

Acknowledgment. This research was supported by the National Science Foundation via Grant NSF-CHE-03-16896.

\section{References and Notes}

(1) Blokzijl, W.; Engberts, J. B. F. N. Angew. Chem., Int. Ed. Engl. 1993, 32, 1545-1579.

(2) Gill, S. J.; Wadsö, I. Proc. Natl. Acad. Sci. U.S.A. 1976, 73, 29552958.

(3) Muller, N. Acc. Chem. Res. 1990, 23, 23-28.

(4) Kronberg, B.; Costas, M.; Silveston, R. Pure Appl. Chem. 1995, $67,897-902$.

(5) Lüdemann, S.; Abseher, R.; Schreiber, H.; Steinhauser, O. J. Am. Chem. Soc. 1997, 119, 4206-4213.

(6) Paschek, D. J. Chem. Phys. 2004, 120, 6674-6690.

(7) Edsall, J. T. J. Am. Chem. Soc. 1935, 57, 1506-1507.

(8) Eley, D. D. Trans. Faraday Soc. 1939, 35, 1281-1293.

(9) Eley, D. D. Trans. Faraday Soc. 1939, 35, 1421-1432.

(10) Paschek, D. J. Chem. Phys. 2004, 120, 10605-10617.

(11) Privalov, P. L.; Gill, S. J. Adv. Protein Chem. 1988, 39, 191-234.

(12) Ray, C.; Brown, J. R.; Akhremitchev, B. B. J. Phys. Chem. B 2006

$110,17578-17583$

(13) Guillot, B.; Guissani, Y. J. Chem. Phys. 1993, 99, 8075-8094.

(14) Garde, S.; Hummer, G.; García, A. E.; Paulaitis, M. E.; Pratt, L. R.

Phys. Rev. Lett. 1996, 77, 4966-4968.

(15) Dill, K. A. Biochemistry 1990, 29, 287-297.

(16) Dill, K. A. Science (Washington, DC, U.S.) 1990, 250, 297.

(17) Butler, J. A. V. Trans. Faraday Soc. 1937, 33, 229-238.

(18) Frank, H. S.; Evans, M. W. J. Chem. Phys. 1945, 13, 507-532.

(19) Lee, B. Proc. Natl. Acad. Sci. U.S.A. 1991, 88, 5154-5158.

(20) Baldwin, R. L. Proc. Natl. Acad. Sci. U.S.A. 1986, 83, 8069-8072.

(21) Shinoda, K. J. Phys. Chem. 1977, 81, 1300-1302.

(22) Kauzmann, W. Adv. Protein Chem. 1959, 14, 1-63.

(23) Nemethy, G.; Scheraga, H. A. J. Chem. Phys. 1962, 36 (12), 34013417.

(24) Gill, S. J.; Dec, S. F.; Olofsson, G.; Wadsoe, I. J. Phys. Chem. 1985, 89, 3758-3761.

(25) Stillinger, F. H. J. Solution Chem. 1973, 2, 141-158.

(26) Geiger, A.; Rahman, A.; Stillinger, F. H. J. Chem. Phys. 1979, 70, 263-276.

(27) Rajamani, S.; Truskett, T. M.; Garde, S. Proc. Natl. Acad. Sci. U.S.A. 2005, 102, 9475-9480.

(28) Zangi, R.; Hagen, M.; Berne, B. J. J. Am. Chem. Soc. 2007, 129, $4678-4686$.

(29) Wallqvist, A.; Berne, B. J. J. Phys. Chem. 1995, 99, 2893-2899. (30) Huang, X.; Margulis, C. J.; Berne, B. J. Proc. Natl. Acad. Sci. U.S.A 2003, 100, 11953-11958.

(31) Lum, K.; Chandler, D.; Weeks, J. D. J. Phys. Chem. B 1999, 103, $4570-4577$.

(32) Chandler, D. Nature (London, U.K.) 2002, 417, 491.

(33) Chandler, D. Nature (London, U.K.) 2005, 437, 640-647.

(34) Zangi, R.; Berne, B. J. J. Phys. Chem. B 2006, 110, 22736-22741.

(35) Privalov, P. L.; Makhatadze, G. I. J. Mol. Biol. 1993, 232, 660679 .

(36) Sturtevant, J. M. Proc. Natl. Acad. Sci. U.S.A. 1977, 74, 22362240

(37) Fu, L.; Freire, E. Proc. Natl. Acad. Sci. U.S.A. 1992, 89, 93359338.

(38) Huang, D. M.; Chandler, D. Proc. Natl. Acad. Sci. U.S.A. 2000 97, 8324-8327.

(39) Robertson, A. D.; Murphy, K. P. Chem. Rev. 1997, 97, 1251-1267.

(40) Yushchenko, V. S.; Yaminsky, V. V.; Shchukin, E. D. J. Colloid Interface Sci. 1983, 96, 307-314.

(41) Bérard, D. R.; Attard, P.; Patey, G. N. J. Chem. Phys. 1993, 98 , $7236-7244$

(42) Huang, X.; Zhou, R.; Berne, B. J. J. Phys. Chem. B 2005, 109, $3546-3552$
(43) Huang, X.; Margulis, C. J.; Berne, B. J. J. Phys. Chem. B 2003, 107, 11742-11748.

(44) Zhou, R.; Huang, X: Margulis, C. J.; Berne, B. J. Science (Washington, DC, U.S.) 2004, 305, 1605-1609.

(45) Liu, P.; Huang, X.; Zhou, R.; Berne, B. J. Nature (London, U.K.) $\mathbf{2 0 0 5}, 437,159-162$

(46) Luzar, A.; Leung, K. J. Chem. Phys. 2000, 113 (14), 5836-5844.

(47) Leung, K.; Luzar, A. J. Chem. Phys. 2000, 113 (14), 5845-5852.

(48) Leung, K.; Luzar, A.; Bratko, D. Phys. Rev. Lett. 2003, 90 (6), 65502-1-065502-4.

(49) Berendsen, H. J. C.; Grigera, J. R.; Straatsma, T. P. J. Phys. Chem. 1987, 91, 6269-6271.

(50) Boulougouris, G. C.; Economou, I. G.; Theodorou, D. N. J. Phys. Chem. B 1998, 102, 1029-1035.

(51) Li, X.; Li, J.; Eleftheriou, M.; Zhou, R. J. Am. Chem. Soc. 2006, 128 (38), 12439-12447.

(52) Lindahl, E.; Hess, B.; van der Spoel, D. J. Mol. Model. 2001, 7, 306-317. 962.

(54) Berendsen, H. J. C.; Postma, J. P. M.; van Gunsteren, W. F.; DiNola, A.; Haak, J. R. J. Chem. Phys. 1984, 81, 3684-3690.

(55) Pangali, C. S.; Rao, M.; Berne, B. J. Computer Modeling of Matter; Lykos, P., Ed.; ACS Symposium Series No. 86; ACS: Washington, DC, 1978; p 29

(56) Watanabe, K.; Andersen, H. C. J. Phys. Chem. 1986, 90, 795802.

(57) Flyvbjerg, H.; Petersen, H. G. J. Chem. Phys. 1989, 91, 461-466.

(58) Nosé, S. J. Chem. Phys. 1984, 81, 511-519.

(59) Hoover, W. G. Phys. Rev. A: At., Mol., Opt. Phys. 1985, 31, 16951697.

(60) Werder, T.; Walther, J.; Jaffe, R.; Halicioglu, T.; Koumoutsakos, P. J. Phys. Chem. B 2003, 107, 1345-1352.

(61) Hirvi, J. T.; Pakkanen, T. A. J. Chem. Phys. 2006, 125, 1447121-144712-11.

(62) Garde, S.; García, A. E.; Pratt, L. R.; Hummer, G. Biophys. Chem. 1999, 78, 21-32.

(63) Choudhury, N.; Pettitt, B. M. J. Phys. Chem. B 2006, 110 (16), 8459-8463.

(64) Murphy, K. P.; Privalov, P. L.; Gill, S. J. Science (Washington, DC, U.S.) 1990, 247, 559-561.

(65) Pauling, L. J. Am. Chem. Soc. 1935, 57, 2680-2684.

(66) Lee, C. Y.; McCammon, J. A.; Rossky, P. J. J. Chem. Phys. 1984, $80,4448-4455$.

(67) Zangi, R.; Engberts, J. B. F. N. J. Am. Chem. Soc. 2005, 127, 22722276.

(68) Hore, D. K.; Walker, D. S.; MacKinnon, L.; Richmond, G. L. J. Phys. Chem. C 2007, 111, 8832-8842.

(69) Masterton, W. L. J. Chem. Phys. 1954, 22, 1830-1833.

(70) Widmer-Cooper, A.; Harrowell, P. J. Non-Cryst. Solids 2006, 352, $5098-5102$

(71) Liu, P.; Harder, E.; Berne, B. J. J. Phys. Chem. B 2004, 108, 65956602

(72) Hua, L.; Huang, X.; Zhou, R.; Berne, B. J. J. Phys. Chem. B 2006, $110,3704-3711$.

(73) Schonhorn, H. J. Phys. Chem. 1966, 70, 4086-4087.

(74) Phillips, M. C.; Riddiford, A. C. Nature (London, U.K.) 1965, 205, 1005-1006.

(75) Petke, F. D.; Ray, B. R. J. Colloid Interface Sci. 1969, 31, 216227.

(76) Jones, J. B.; Adamson, A. W. J. Phys. Chem. 1968, 72, 646-650.

(77) Li, Z.; Liang, X.; Zhou, Y.; Tang, J.; Cui, J.; Liu, Y. Annual Report Conference on Electrical Insulation and Dielectric Phenomena; 2004; pp $679-682$.

(78) Adam, N. K. Advanced Chemistry Series; American Chemical Society: Washington, DC, 1964; Vol. 43, pp 52-56.

(79) Hill, T. L. Thermodynamics of Small Systems; Dover Publications: Phoenix, 2002.

JP802135C 